\title{
Stability of strain gradient elastic bars in tension
}

\author{
D. G. Bakogianni · K. A. Lazopoulos
}

Published online: 21 March 2008

(C) Springer-Verlag 2008

\section{Erratum to: Optimization Letters (2007) 1:407-420 DOI:10.1007/s11590-006-0039-9}

The error appears in the value of $\omega$ defined by Eq. (33a) of the text. In fact with the selected values of $c_{1}, c_{2}, c_{3}, \omega$ is not real. Hence the values of $\mathrm{c}_{1}, \mathrm{c}_{2}, \mathrm{c}_{3}$ should be changed and the section of "Application" should be modified. The corrected part follows.

\section{Application}

Implementing the proposed theory, we consider an application with the second gradient unidirectional elastic moduli,

$$
c_{1}=-6.75 \times 10^{-2} \mathrm{~cm}^{2}, \quad c_{2}=1.0 \times 10^{-3} \mathrm{~cm}^{4}, \quad c_{3}=-1 \times 10^{-3} \mathrm{~cm}^{2} .
$$

The critical load defined by Eq. (33b) has been found equal to $p_{o}=1.07133$ and the mode number $\omega=57.21 \mathrm{~cm}^{2}$ computed by Eq. (33a). Further, the large homogeneous displacement parameter $\alpha_{o}=0.08$ may be defined through Eq. (19). Likewise, the critical first Piola stress $\sigma_{o}=0.09$ is computed recalling Eq. (11). The application

The online version of the original article can be found under doi:10.1007/s11590-006-0039-9.

D. G. Bakogianni · K. A. Lazopoulos $(\varangle)$

Mechanics Division, School of Applied Sciences, National Technical University of Athens, Zografou Campus, 15773 Athens, Greece

e-mail: kolazop@central.ntua.gr 


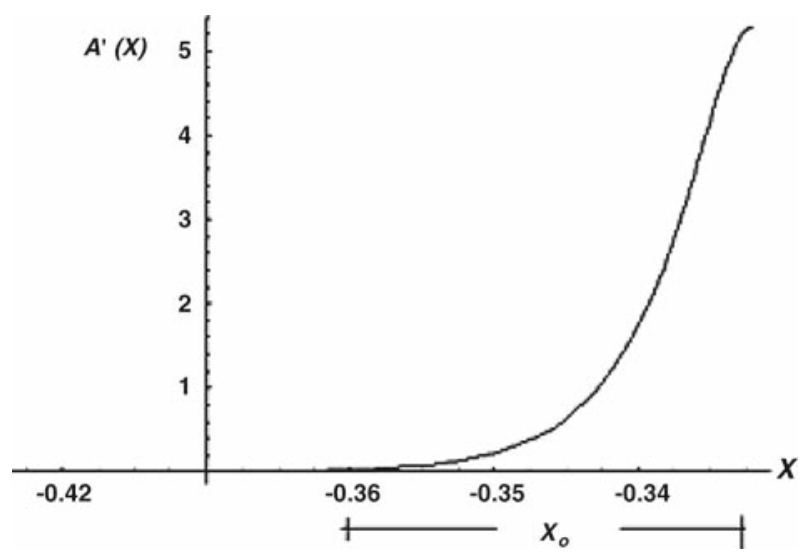

Fig. 2 The strain localization for the application

is performed with the incremental loading parameter $\gamma=5$, see Eq. (12). For the present problem Eq. (41) becomes,

$$
\frac{\partial A_{R}(X)}{\partial X}=\left(1.043 \times 10^{4} A_{R}^{2}(X)-1.945 \times 10^{6} A_{R}^{4}(X)\right)^{1 / 2}
$$

Inversing Eq. (43) and integrating we get, $X=30491\left(3.21 \times 10^{-7}\left(\log \left[A^{2}\right]-\right.\right.$ $\left.\left.\log \left[9.66 \times 10^{12} A+3.17 \times 10^{6} \sqrt{9.66 \times 10^{12} A^{2}-9.02 \times 10^{14} A^{4}}\right]\right)\right)$ for $0<A<$ 0.073 . The region of integration is defined by Eq. (40), recalling the strain is maximum at the middle of the bar.

The localization of the strain is presented in Fig. 2. 